\title{
The X-ray properties of the magnetic cataclysmic variable UU Columbae ${ }^{\star}$
}

\author{
D. de Martino ${ }^{1}$, G. Matt ${ }^{2}$, K. Mukai ${ }^{3}$, J.-M. Bonnet-Bidaud ${ }^{4}$, \\ V. Burwitz ${ }^{5}$, B. T. Gänsicke ${ }^{6}$, F. Haberl ${ }^{5}$, and M. Mouchet ${ }^{7}$
}

1 INAF-Osservatorio Astronomico di Capodimonte, via Moiariello 16, 80131 Napoli, Italy

e-mail: demartino@na.astro.it

2 Dipartimento di Fisica, Universita' degli Studi Roma Tre, via della Vasca Navale 84, 00146 Roma, Italy e-mail: matt@fis.uniroma3.it

3 Laboratory for High Energy Astrophysics, NASA/GSFC, Code 662, Greenbelt, MD 20771, USA

e-mail: mukai@milkyway.gsfc.nasa.gov

${ }^{4}$ Service d'Astrophysique, DSM/DAPNIA/SAp, CE Saclay, 91191 Gif-sur-Yvette Cedex, France e-mail: bonnetbidaud@cea.fr

5 Max-Planck-Institut für Extraterrestrische Physik, Giessenbachstraße, Postfach 1312, 85741 Garching, Germany e-mail: [burwitz; fwh]@mpe.mpg.de

${ }^{6}$ Department of Physics, University of Warwick, Coventry CV4 7AL, UK

e-mail: boris .gaensicke@warwick.ac.uk

7 APC, UMR 7164, University Denis Diderot, 2 place Jussieu, 75005 Paris and LUTH, Observatoire de Paris, 92195 Meudon Cedex, France

e-mail: martine.mouchet@obspm.fr

Received 23 February 2006 / Accepted 11 March 2006

ABSTRACT

\begin{abstract}
Aims. XMM-Newton observations to determine for the first time the broad-band X-ray properties of the faint, high galactic latitude intermediate polar UU Col are presented.

Methods. We performed X-ray timing analysis in different energy ranges of the EPIC cameras, which reveals the dominance of the $863 \mathrm{~s}$ white dwarf rotational period. The spin pulse is strongly energy dependent. Weak variabilities at the beat $935 \mathrm{~s}$ and at the $3.5 \mathrm{~h}$ orbital periods are also observed, but the orbital modulation is detected only below $0.5 \mathrm{keV}$. Simultaneous UV and optical photometry shows that the spin pulse is anti-phased with respect to the hard X-rays. Analysis of the EPIC and RGS spectra reveals the complexity of the X-ray emission, which is composed of a soft $50 \mathrm{eV}$ black-body component and two optically thin emission components at $0.2 \mathrm{keV}$ and $11 \mathrm{keV}$ strongly absorbed by dense material with an equivalent hydrogen column density of $10^{23} \mathrm{~cm}^{-2}$ that partially $(50 \%)$ covers the X-ray source.

Results. The complex X-ray and UV/optical temporal behaviour indicates that accretion occurs predominantly $(\sim 80 \%)$ via a disc with a partial contribution $(\sim 20 \%)$ directly from the stream. The main accreting pole dominates at high energies whilst the secondary pole mainly contributes in the soft X-rays and at lower energies. The bolometric flux ratio of the soft-to-hard X-ray emissions is found to be consistent with the prediction of the standard accretion shock model. We find the white dwarf in UU Col accretes at a low rate and possesses a low magnetic moment. It is therefore unlikely that UU Col will evolve into a moderate field strength polar, so that the soft X-ray intermediate polars still remain an enigmatic small group of magnetic cataclysmic variables.
\end{abstract}

Key words. stars: binaries: close - stars: individual: RX J0512.2-3241 (UU Col) - stars: novae, cataclysmic variables $\mathrm{X}$-rays: binaries - accretion, accretion disks

\section{Introduction}

The intermediate polars (IPs), a subclass of magnetic cataclysmic variables (mCVs), are the most luminous and hardest $\mathrm{X}$-ray sources among accreting white dwarfs (WDs). They contain a magnetized asynchronously rotating $\mathrm{WD}\left(P_{\omega} \ll P_{\Omega}\right)$ accreting from a late type, Roche-lobe filling star, usually via a truncated disc. Accretion falls quasi-radially towards the magnetic poles, forming a standing shock below which material cools via hard $\mathrm{X}$-rays and cyclotron emission.

* Based on observations obtained with XMM-Newton, an ESA science mission with instruments and contributions directly funded by ESA Member States and NASA.
IPs differ from the polars, the other subclass of mCVs, which instead are synchronous, strong emitters of soft X-rays, as well as of optical/near-IR polarized radiation. Due to the intense (10-230 MG) WD magnetic field, the polars do not possess an accretion disc. The soft X-ray emission is believed to arise from the reprocessing of hard X-rays and cyclotron radiation in the WD atmosphere. The ratio of hard to soft X-ray fluxes strongly depends on the magnetic field strength, reflecting the interplay between thermal bremsstrahlung and cyclotron cooling, with the latter dominating in high field systems (Woelk \& Beuermann 1992). The lack of soft X-ray emission in the majority of IPs was explained by the higher accretion rates, the high intrinsic absorption, and the larger accreting areas with respect to the polars shifting the peak of reprocessed emission to the EUV/UV range. 
Table 1. Summary of the XMM-Newton observation of UU Col.

\begin{tabular}{|c|c|c|c|c|}
\hline Instrument & Date & UT(start) & Duration (s) & Net count rate $\left(\right.$ cts s $\left.^{-1}\right)$ \\
\hline EPIC PN & 2004 Aug. 21 & $22: 00$ & 26037 & 0.50 \\
\hline EPIC MOS & & $21: 38$ & 27666 & 0.18 \\
\hline RGS & & $21: 37$ & 27918 & 0.018 \\
\hline \multirow[t]{5}{*}{ OM B } & & $21: 46$ & 2441 & 3.10 \\
\hline & & $22: 32$ & 2441 & \\
\hline & & $23: 18$ & 2440 & \\
\hline & 2004 Aug. 22 & 00:04 & 2440 & \\
\hline & & $00: 50$ & 2439 & \\
\hline \multirow[t]{5}{*}{ OM UVM2 } & & $01: 36$ & 2439 & 0.47 \\
\hline & & 02:22 & 2440 & \\
\hline & & 03:08 & 2440 & \\
\hline & & 03:54 & 2439 & \\
\hline & & $4: 40$ & 2439 & \\
\hline
\end{tabular}

The absence of polarized radiation in the optical and near-IR in the majority of these systems also led to the suggestion that they possess lower magnetic field strength WDs than the polars.

Whether IPs will evolve into polars or they represent a distinct class is still a debated evolutionary issue (Cumming 2002; Norton et al. 2004). A small group of four IPs (PQ Gem, V405 Aur, UU Col, and 1RXS J062518.2+733433) was recognized to also possess a soft X-ray emission component (Haberl \& Motch 1995; Burwitz et al. 1996; Staude et al. 2003), similar to the one observed in polars with two optically bright systems (PQ Gem and V405 Aur) also showing optical polarized radiation. Their similarity to low field polars led to the suggestion that these IPs (also called "soft IPs") could be their true progenitors. Recently, two hard X-ray IPs were discovered to possess a soft $\mathrm{X}$-ray component but were also found to be highly absorbed and hotter than that of the soft IPs and polars (Haberl et al. 2002; de Martino et al. 2004), raising the question of whether a soft $\mathrm{X}$-ray component is indeed present in all IPs.

Since its discovery from the ROSAT All Sky Survey (RASS), the high latitude X-ray source 1RXS J0512.2-3241=UUCol (henceforth UU Col), identified as a soft X-ray IP (Burwitz et al. 1996), mostly went unnoticed. A follow-up X-ray ROSAT HRI pointed observation (Burwitz \& Reinsch 2001) confirmed the optical photometric periods of $3.45 \mathrm{~h}$ and $863.5 \mathrm{~s}$ interpreted as the binary period and the rotational period of the accreting WD, respectively. However, a knowledge of its broad band Xray properties had to await the advent of more sensitive X-ray facilities.

In this work we report on the first XMM-Newton observation of UUCol that were aimed at determining the variability characteristics in both X-ray and UV/optical domains, as well as the X-ray spectral properties, of this poorly studied $\mathrm{mCV}$ and at inferring the accretion and WD parameters to understand its evolutionary state.

\section{The XMM-Newton observation}

UU Col was observed with the XMM-Newton satellite (Jansen et al. 2001) on August 21, 2004 (obsid:0201290201) with the EPIC-PN (Strüder et al. 2001) and MOS (Turner et al. 2001) cameras operated in full frame mode with the thin filter for a net exposure time of $26.0 \mathrm{ks}$ and $27.7 \mathrm{ks}$, respectively. UU Col was also observed with the Reflection Grating Spectrographs (RGS1 and RGS2) (den Herder et al. 2001) in spectroscopy mode with an exposure time of $27.9 \mathrm{ks}$ and with the Optical Monitor (OM) instrument (Mason et al. 2001) with the UVM2 and $B$ filters covering the ranges $2000-2800 \AA$ and $3900-4900 \AA$ in imaging fast mode for a total exposure time of $12.3 \mathrm{ks}$ in each filter. A summary of the observations is reported in Table 1.

The standard processing pipeline data products were used. The EPIC light curves and spectra and the RGS spectra were extracted with the SAS 6.5 package retrieved from the ESA-VILSPA Science Center. Due to the proximity of UU Col to the CCD No. 1 border of the EPIC PN camera, the light curves and spectra were extracted from a circular region with a radius of $17.5^{\prime \prime}$ centred on the source, while for the EPIC MOS cameras a larger extraction radius $40^{\prime \prime}$ was used. Background light curves and spectra were extracted from offset circular regions with the same radii as for the target on the same CCD chip. Single and double pixel events with a zero quality flag were selected for the EPIC-PN data, while up to quadruple pixel events were used for EPIC-MOS cameras.

The EPIC-PN and, to a less extent, the MOS data, are affected by only moderate background activity (up to $0.16 \mathrm{cts} \mathrm{s}^{-1}$ ) not influencing the light curve (see Fig. 1). However, for the spectral analysis, we conservatively windowed the data in order to exclude epochs when background count rate exceeds $0.13 \mathrm{cts} \mathrm{s}^{-1}$ in the EPIC-PN camera, implying a $\sim 20 \%$ screening of the data. The extracted EPIC-PN and MOS average spectra were then rebinned to have a minimum of 20 counts in each bin, while phase-resolved spectra were rebinned with a minimum of 25 counts per bin to allow the use of the $\chi^{2}$ statistics. Ancillary response and redistribution matrix files were created using SAS tasks arfgen and rmfgen, respectively.

The RGS pipeline was run using the SAS task rgsproc. RGS1 and RGS2 first-order spectra have been rebinned to have a minimum of 20 counts per bin.

The OM background subtracted light curves produced by the standard processing pipeline were used for timing analysis (see Fig. 1). Average net count rates are $3.106 \mathrm{cts} \mathrm{s}^{-1}$ ( $B$ filter) and $0.471 \mathrm{cts} \mathrm{s}^{-1}$ (UVM2 filter), corresponding to the instrumental magnitudes: $B=18.0 \pm 0.6 \mathrm{mag}$ and $U V M 2=16.6 \pm 0.7 \mathrm{mag}$. Using Vega magnitude-to-flux conversion, these correspond to a flux of $3.9 \times 10^{-16} \mathrm{erg} \mathrm{cm}^{-2} \mathrm{~s}^{-1} \AA^{-1}$ in the $3900-4900 \AA$ band and of $1.02 \times 10^{-15} \mathrm{erg} \mathrm{cm}^{-2} \mathrm{~s}^{-1} \AA^{-1}$ in the $2000-2800 \AA$ band. The $B$ band level is consistent with that observed in 1996 by Burwitz et al. (1996).

\section{Timing analysis}

We performed a search for periodic variations in both X-ray and UV/optical data in order to characterize the temporal behaviour of UU Col on a wide energy range. 


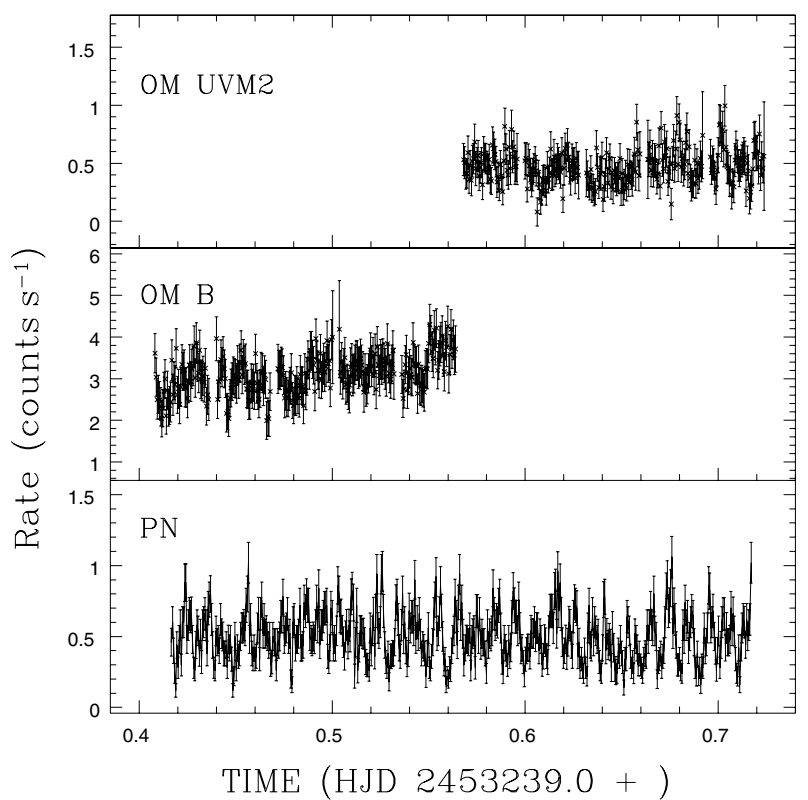

Fig. 1. From bottom to top: the light curves in the EPIC PN $0.2-15 \mathrm{keV}$ range, in the $B$ band, and in the UV 2000-2800 ̊ range binned in $50 \mathrm{~s}$.

\subsection{The X-ray light curves and power spectra}

A search for variability was performed by extracting X-ray light curves from the all available channels of EPIC PN and MOS cameras, i.e. between $0.2-15 \mathrm{keV}$ range with a $50 \mathrm{~s}$ time resolution (see Fig. 1). A periodic modulation is apparent and confirmed by a Fourier analysis of the light curve, shown in Fig. 2 for the PN camera. Strong power is found at 100 day $^{-1}$, while weaker peaks are detected at its second harmonic and at $\sim 92$ day $^{-1}$. The former corresponds to the optical pulse period found by Burwitz et al. (1996) and the latter to the beat (the orbital sideband $\omega-\Omega$ ) that was detected only at optical wavelengths. No sign of low-frequency variability is detected in the whole $0.2-15 \mathrm{keV}$ EPIC band.

We then performed a sinusoidal fit to the EPIC light curves, including four sinusoids accounting for the fundamental, the first, and second harmonic and for the beat periods. This gives $\omega=100.08 \pm 0.16 \mathrm{day}^{-1}$ and $\omega-\Omega=92.37 \pm 0.39 \mathrm{day}^{-1}$ for the PN light curve, while for the MOS we find $\omega=99.86 \pm$ $0.24 \mathrm{day}^{-1}$ and $\omega-\Omega=93.74 \pm 0.60 \mathrm{day}^{-1}$. Because of the higher-fit accuracy of the PN data, we then adopt $P_{\omega}=863.3 \pm$ $1.4 \mathrm{~s}$ for the spin period. The time of maximum of the X-ray spin pulse is then: $\mathrm{HJD}_{\max }=2453239.5648(1)+0.00999(2) \mathrm{E}$. The beat frequency implies an orbital period of $P_{\Omega}=3.13 \pm 0.17 \mathrm{~h}$, broadly consistent with the optical determination by Burwitz et al. (1996). The amplitude of the beat variability is relatively low $\sim 9 \%$.

A Fourier analysis has also been performed on light curves extracted with the same $50 \mathrm{~s}$ bin time in selected energy bands, $0.2-0.5 \mathrm{keV}, 0.5-1 \mathrm{keV}, 1-2 \mathrm{keV}, 2-5 \mathrm{keV}$ and $5-12 \mathrm{keV}$ as reported in Fig. 2. A different behaviour between soft and hard ranges is observed, the second harmonic dominating the softest range $(0.2-0.5 \mathrm{keV})$ with an indication of a low frequency periodicity at $9.2 \mathrm{day}^{-1}$, while the spin frequency dominates between $0.5-5 \mathrm{keV}$. No variability is detected above $5 \mathrm{keV}$.

Using the determined spin ephemeris, we folded the light curves in the different energy ranges as shown in Fig. 3, as well as the hardness ratios defined as the ratio of countrates in [5-12 keV] and [2-5 keV] ranges, in [2-5 keV] and [1-2 keV],

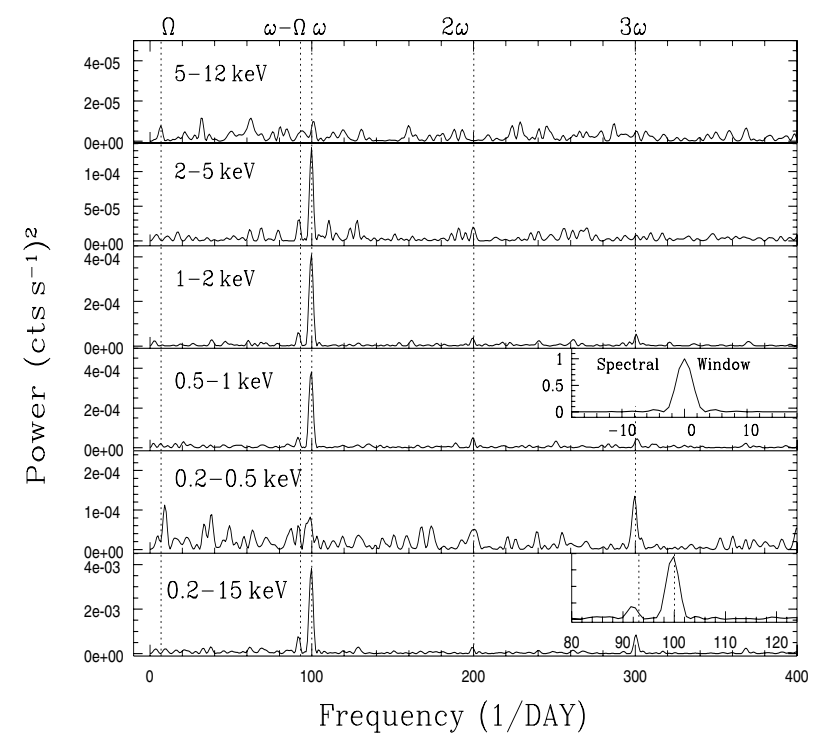

Fig. 2. EPIC-PN power spectra in selected energy ranges. From bottom to top: $0.2-15 \mathrm{keV}, 0.2-0.5 \mathrm{keV}, 0.5-1 \mathrm{keV}, 1-2 \mathrm{keV}, 2-5 \mathrm{keV}$, and $5-12 \mathrm{keV}$. The spin $(\omega)$ and harmonics, the beat $(\omega-\Omega)$, and the orbital $(\Omega)$ frequencies as derived from optical data (Burwitz et al. 1996) are marked with vertical dotted lines. Inserted panels show an enlargement around the spin frequency (lower panel) and the spectral window of the data.

in the $[1-2 \mathrm{keV}]$ and $[0.5-1 \mathrm{keV}]$ ranges, in the $[0.5-1 \mathrm{keV}]$ and $[0.2-0.5 \mathrm{keV}]$ ranges. While no significant modulation is detected above $5 \mathrm{keV}$, a sawtooth-like spin pulse is observed in the $0.5-5 \mathrm{keV}$ range, with fractional amplitudes (half-amplitude) of $27 \pm 2 \%[2-5 \mathrm{keV}], 37 \pm 2 \%[1-2 \mathrm{keV}]$, and $33 \pm 2 \%$ [0.5-1 keV]. A dip centred on the maximum of the pulsation is observed more pronounced between $0.5-2 \mathrm{keV}$. This feature has also been observed in V709 Cas (Norton et al. 1999). From the hardness ratios the pulse hardens at spin minimum between $1-12 \mathrm{keV}$, while it shows no energy dependence between $0.5-2 \mathrm{keV}$. Below $0.5 \mathrm{keV}$ and as indicated by the power spectra, the spin modulation instead shows approximately three maxima, produced by the dip centred on the primary maximum seen in the harder bands and an additional maximum appearing at the minimum of the hard band pulse. The appearance of this maximum is clearly seen in the hardness ratios where an antiphase behaviour is observed with respect to the hard bands.

The length of the EPIC coverage is about twice the $3.5 \mathrm{~h}$ orbital period detected in the optical (Burwitz et al. 1996) and ROSAT data (Burwitz \& Reinsch 2001). A sinusoidal fit to the PN light curve in the $0.2-0.5 \mathrm{keV}$ band prewhitened from the high frequency spin variability gives an orbital period of $3.55 \pm 0.56 \mathrm{~h}$, thus confirming an orbital modulation in the soft X-ray band. The folded orbital light curve reported in Fig. 4 shows a modulation with a fractional amplitude of $\sim 10 \%$, similar in shape but much weaker than found in the ROSAT HRI data (Burwitz \& Reinsch 2001). We also explored the orbital dependence of the soft X-ray emission and, in particular, the dependence of the soft X-ray spin pulse on the orbital cycle. We then extracted the spin light curves in the $0.2-0.5 \mathrm{keV}$ range at maximum and minimum of the orbital modulation, i.e. between $\phi_{\Omega}=0.4 \div 0.7$ and $\phi_{\Omega}=-0.2 \div+0.2$ (a finer binning does not provide good statistics of light curves). Figure 5 shows that the pulse profile changes with the orbital phase, since it is similar to the orbital phase-average spin pulse profile at orbital minimum, whilst at orbital maximum, the spin pulse shows a peak 

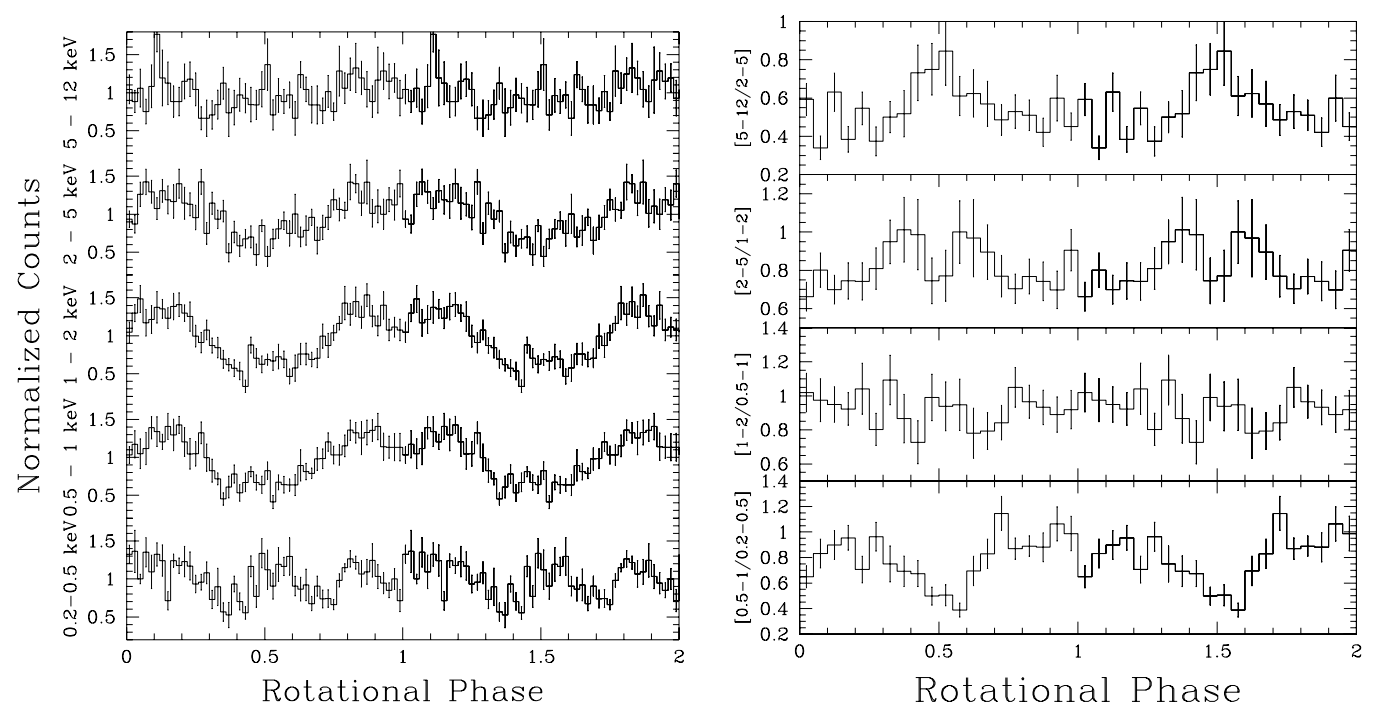

Fig. 3. Left: EPIC-PN folded light curves in selected energy bands at the $863.3 \mathrm{~s}$ period using the ephemeris quoted in the text. Right: the EPIC hardness ratios show the complex energy dependence of the pulse.

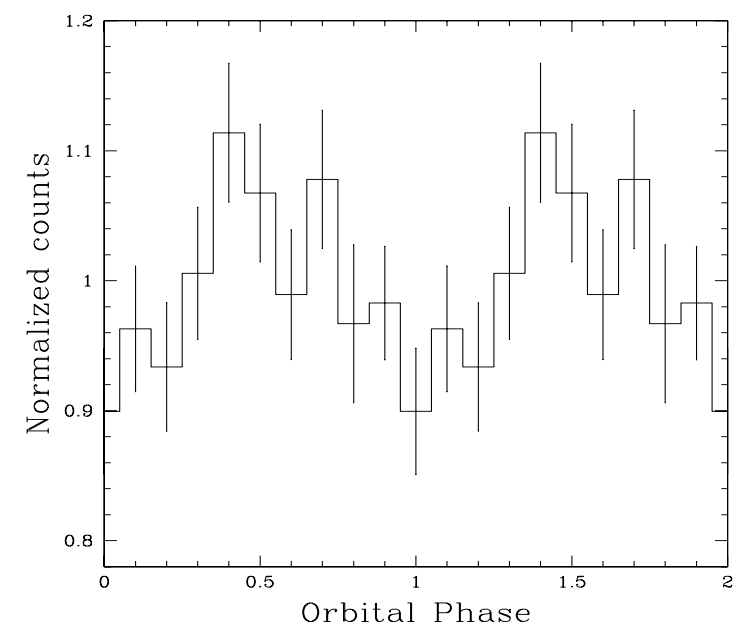

Fig. 4. The $0.2-0.5 \mathrm{keV}$ EPIC PN folded light curve at the $3.55 \mathrm{~h}$ orbital period.

at $\phi_{\omega} \sim 0.5$, i.e. at the minimum of the hard X-ray spin pulse. This behaviour implies that the orbital modulation in the soft Xrays is dominated by a component that is anti-phased and hence not linked to the hard X-ray emission. This also explains the lack of an orbital periodicity at high energies.

\subsection{The UV and optical light curves}

The $U V M 2$ and $B$ band light curves shown in Fig. 1 reveal a short-term modulation, but given the short time coverage of the OM observations in the two bands, we limit ourselves to folding the light curves at the determined WD spin period (Fig. 6). These show an amplitude modulation of $15 \%$ and $6 \%$ in the UV and optical band, suggesting a hot component responsible for the pulsation at these wavelengths. The $B$ band pulse has similar amplitude to the one observed by Burwitz et al. (1996). The advantage of simultaneity allows us to compare X-ray and UV/optical rotational modulations. In contrast to many IPs, UU Col shows an antiphased behaviour, with the minimum of UV and optical pulses centred on the hard X-ray maximum. The UV and optical rotational light curves also do not resemble the soft

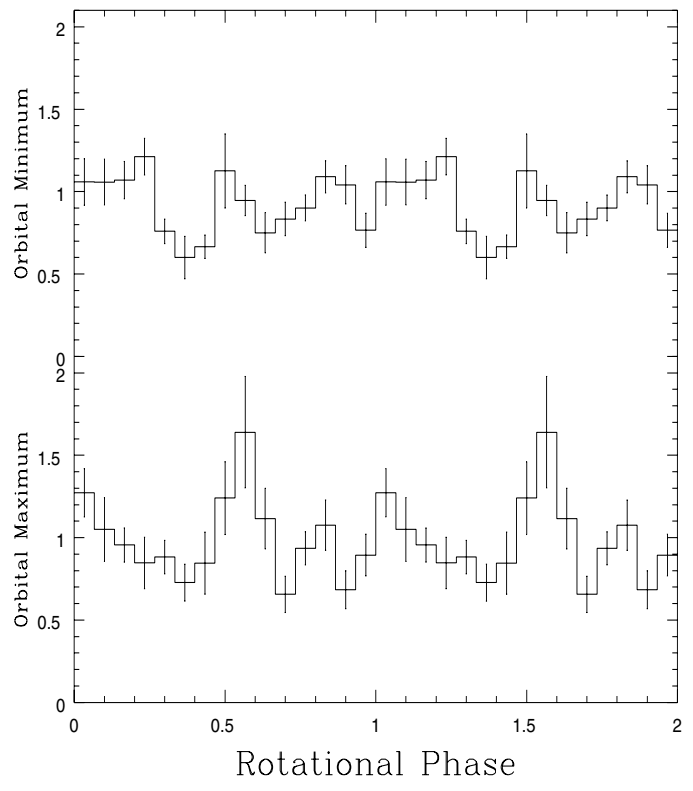

Fig. 5. The $0.2-0.5 \mathrm{keV}$ spin light curves extracted at orbital maximum (lower panel) and orbital minimum (upper panel).

$(0.2-0.5 \mathrm{keV})$ pulse closely implying that the latter is composed of more than one component. However, the maximum of the $\mathrm{UV} /$ optical spin pulse is very broad and centred at $\phi_{\omega}=0.5$ where the soft band also shows a maximum. This indicates that at least part of the soft X-rays are produced in a region that is somewhat linked to the one producing the UV and optical pulsations. The wider phase range of the UV/optical pulse, however, implies a wider emitting area.

\section{The complex X-ray spectrum of UU Col}

Identification of spectral components was performed on the EPIC PN and the combined MOS-averaged spectra using the XSPEC package. Due to calibration-accuracy issues, the spectra were analysed between 0.3 and $10 \mathrm{keV}$ (Fig. 7). A simple model consisting of an optically thin plasma MEKAL $\left(\left(k T_{\mathrm{MK}}=70 \mathrm{keV}\right)\right.$ plus a black-body $\left(k T_{\mathrm{BB}}=80 \mathrm{eV}\right)$ does not satisfactorily 
Table 2. Spectral parameters as derived from simultaneously fitting the EPIC PN and MOS phase-averaged spectra for the two best fit models discussed in the text. Quoted errors refer to the $90 \%$ confidence level for the parameter of interest.

\begin{tabular}{cccccccccccc}
\hline \hline & \multicolumn{2}{c}{ Partial Absorber } & \multicolumn{2}{c}{ Black-body } & \multicolumn{3}{c}{ MEKAL } & \multicolumn{2}{c}{ MEKAL $^{a}$} & $\chi_{v}^{2}\left(\chi^{2} /\right.$ d.o.f. $)$ \\
& $N_{\mathrm{H}}^{b}$ & $\operatorname{Cov}_{\mathrm{F}}{ }^{c}$ & $k T_{\mathrm{BB}}$ & $C_{\mathrm{BB}}^{d}$ & $A_{\mathrm{Z}}^{e}$ & $k T_{1}$ & $C_{1}^{f}$ & $k T_{2}^{g}$ & $C_{2}^{f}$ & $\alpha^{h}$ & \\
& & $\left(10^{23} \mathrm{~cm}^{-2}\right)$ & & $(\mathrm{eV})$ & $\left(10^{-5}\right)$ & & $(\mathrm{keV})$ & $\left(10^{-4}\right)$ & $(\mathrm{keV})$ & $\left(10^{-4}\right)$ & \\
\hline $\mathrm{A}$ & $1.0_{-0.3}^{+0.4}$ & $0.51_{-0.09}^{+0.07}$ & $49.7_{-2.9}^{+5.6}$ & $1.3_{-0.3}^{+0.5}$ & $0.39 \pm 0.16$ & $0.18 \pm 0.02$ & $2.4_{-0.9}^{+1.1}$ & $11_{-2}^{+6}$ & $19.04_{-1.98}^{+0.38}$ & $0.906(522 / 576)$ \\
$\mathrm{B}$ & $0.83_{-0.32}^{+0.51}$ & $0.43_{-0.17}^{+0.15}$ & $51.2 \pm 6.0$ & $1.04_{-0.28}^{+0.63}$ & $0.53_{-0.26}^{+0.71}$ & $0.18_{-0.01}^{+0.02}$ & $1.6_{-1.0}^{+2.0}$ & $27_{-17}^{+\infty}$ & $53.48_{-19.75}^{+68.16}$ & $1.6_{-0.40}^{+2.9}$ & $0.905(520 / 575)$ \\
\hline
\end{tabular}

${ }^{a}$ Second optically thin plasma is a MEKAL (model A) or CEMEKL (model B). ${ }^{b}$ Column density of the partial absorber. ${ }^{c}$ Covering fraction of partial absorber. ${ }^{d}$ Normalization constant of black-body (BBODY) defined as $L_{39} d_{10}^{-2}$, where $L_{39}$ is the luminosity in units of $10^{39} \mathrm{erg} \mathrm{s}^{-1}$ and, $d_{10}$ the distance in units of $10 \mathrm{kpc} .{ }^{e}$ Metal abundance in units of the cosmic value (Anders \& Grevesse 1989) linked for the two thin plasma emissions. ${ }^{f}$ Normalization constant of MEKAL or CEMEKL model defined as EM $\times 10^{-14}\left(4 \pi D^{2}\right)^{-1}$, where EM is the emission measure in $\mathrm{cm}^{-3}$ and $D$ is the distance in $\mathrm{cm} .{ }^{g}$ Maximum temperature in CEMEKL. ${ }^{h}$ Power law index in CEMEKL.

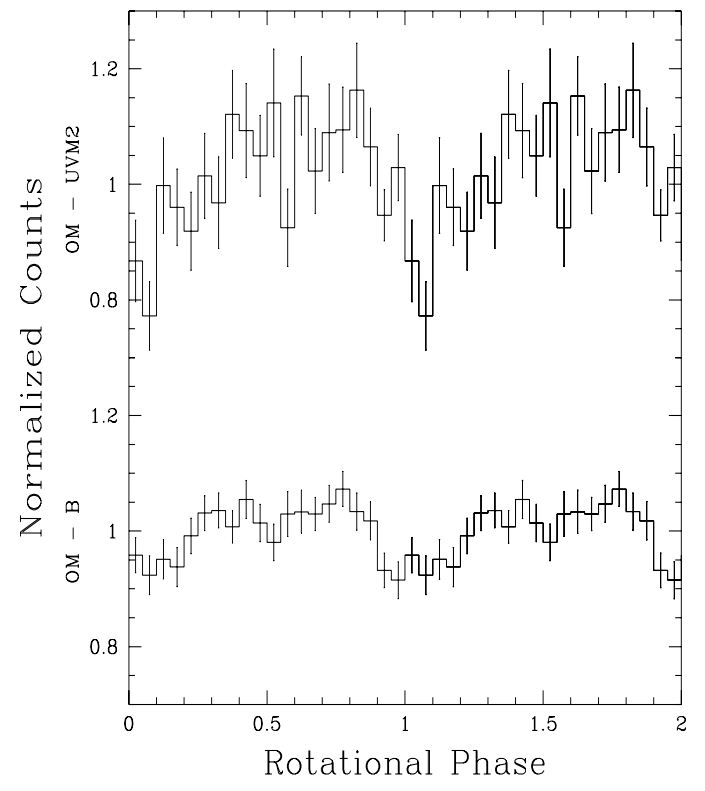

Fig. 6. The UV 2000-2800 AA (upper panel) and the $B$ band spin folded light curves showing the strong colour dependence of pulsation.

$\left(\chi_{v}^{2}=1.10\right)$ reproduce the complex spectrum of UU Col. A dense $\left(N_{\mathrm{H}}=8.3 \times 10^{22} \mathrm{~cm}^{-2}\right)$ partial $(37 \%)$ covering absorber is required $\left(\chi_{v}^{2}=1.04\right)$ and is significant at $99 \%(\mathrm{~F}$-test $)$, which lowers the temperature of the optically thin component to $14 \mathrm{keV}$. The fit further improves $\left(\chi_{v}^{2}=1.00\right)$ when the metal abundance of the optically thin component is left free to vary, giving $A_{\mathrm{Z}}=0.41_{-0.15}^{+0.16}$. Here we note that the derived metal abundance is relative to Anders \& Grevesse (1989) solar abundances. When adopting cosmic abundances as derived for the interstellar medium by Wilms et al. (2000), we find that $A_{\mathrm{Z}}=0.69_{-0.30}^{+0.31}$. This gives a slightly larger hydrogen column density of the partial absorber $\left(N_{\mathrm{H}}=1.5 \times 10^{23} \mathrm{~cm}^{-2}\right)$ but is still consistent within errors with the previous determination.

An excess of counts around $0.6 \mathrm{keV}$ is, however, still present indicating the presence of low temperatures in the post-shock region. Using a multi-temperature model (CEMEKL), where the emission measure varies with the temperature as $T / T_{\max }^{\alpha}$, instead gives a worse fit $\left(\chi_{v}^{2}=1.10\right)$. The best fit is then found by adding another MEKAL component with $k T=0.18 \mathrm{keV}\left(\chi_{v}^{2}=0.906\right)$ (Model A in Table 2). This also lowers the black-body temperature to $50 \mathrm{eV}$, which is more typical of soft X-ray IPs (de Martino et al. 2004). A similar fit quality (Model B) is obtained by substituting the hot optically thin component with a multi-temperature

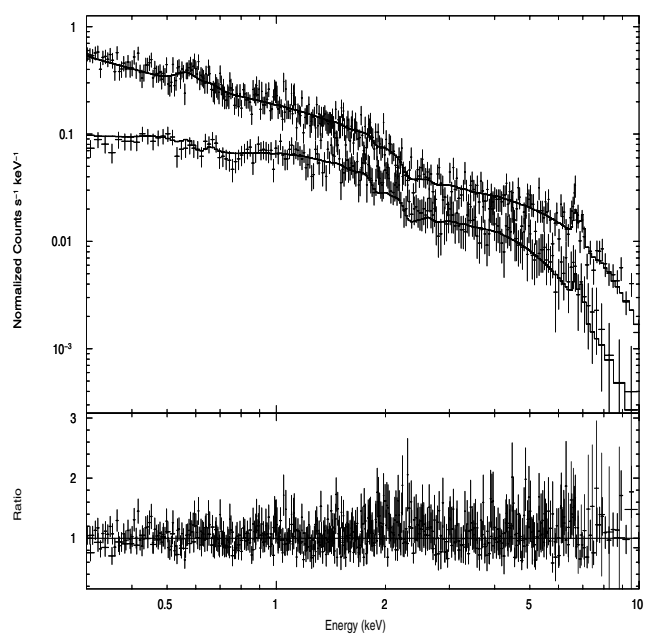

Fig. 7. The EPIC PN (top) and combined MOS (bottom) spectra fitted simultaneously with Model A (see text). The bottom panel shows the ratio between observed and model spectra.

plasma, but many parameters are unconstrained, including an unphysical value for the power law index. An upper limit to the equivalent width of the neutral iron line at $6.4 \mathrm{keV}$ is $76 \mathrm{eV}$. The flux in the $0.2-10 \mathrm{keV}$ range is $1.84 \pm 0.02 \times 10^{-12} \mathrm{erg} \mathrm{cm}^{-2} \mathrm{~s}^{-1}$.

The excess in the $0.6 \mathrm{keV}$ region can also be reproduced by two Gaussians centred at $0.57 \mathrm{keV}$ and $0.65 \mathrm{keV}\left(\chi_{v}^{2}=\right.$ $0.893)$ implying that the O VII $(21.9 \AA)$ He-like triplet and O VIII (19.1 $\AA$ ) $\mathrm{Ly}_{\alpha}$ line are present. Though UUCol is quite faint in the RGS spectra, we can safely detect both lines at $E_{\mathrm{OVII}}=0.570_{-0.021}^{+0.012} \mathrm{keV}$ at a flux $1.5_{-1.2}^{+3.8} \times 10^{-5}$ photons $\mathrm{cm}^{-2} \mathrm{~s}^{-1}$ in the RGS1 and at $E_{\mathrm{OVIII}}=0.648_{-0.006}^{+0.002} \mathrm{keV}$ at a flux $1.2_{-0.6}^{+0.8} \times$ $10^{-5}$ photons $\mathrm{cm}^{-2} \mathrm{~s}^{-1}$ in both RGS. The RGS spectra, when fitted with Model A, give $\chi_{v}^{2}=1.3$ for 46 d.o.f. The large width $(\sim 6 \mathrm{eV})$ of the $\mathrm{O}$ VII line suggests the presence of multiple components. An enlargement of the oxygen region is shown in Fig. 8 rebinning the spectra to have a minimum of 9 counts per bin. We then fitted the O VII line spectral region with two Gaussians and a power law which gives central energies of $0.572 \mathrm{keV}$ and $0.561 \mathrm{keV}$ for these components. The former is broader $(\sigma \sim 2.4 \mathrm{eV})$ and stronger ( 28 times) than the latter. The narrow line at $22.10 \AA$ can be ascribed to the forbidden $(f)$ line component, while the broad feature is probably a blend of the resonance $(r)(21.603 \AA)$ and the intercombination (i) (21.796 $\AA$ ) lines. The relatively large strength of He-like oxygen is consistent with the presence of a low-temperature, 


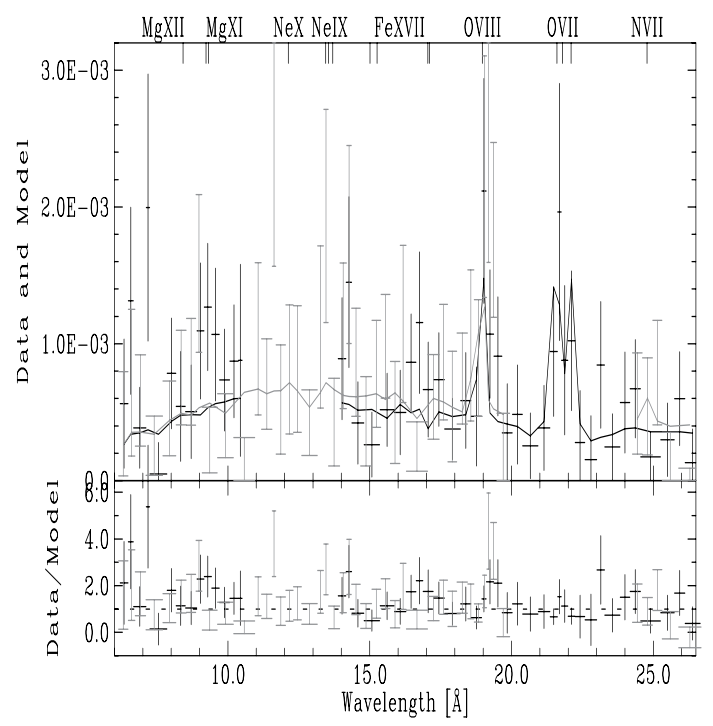

Fig. 8. The RGS spectra (RGS1 in black and RGS2 in gray), along with Model A, derived from the EPIC spectral analysis. The positions of lines expected to be strong are also reported. The ratio between model and observed spectra is shown in the lower panel.

optically thin component. This aspect will be further discussed in Sect. 5 .

In order to identify spectral changes along the spin cycle, we extracted the EPIC PN spectra (the inclusion of MOS spectra does not improve the statistics substantially) at pulse maximum and minimum identified on the total $0.2-15 \mathrm{keV}$ light curve, i.e. between $\phi_{\omega}=-0.2 \div+0.2$ and $\phi_{\omega}=0.3 \div 0.65$, respectively. The spectral fitting was performed using Model A in Table 2, and fixing the metal abundance to the value found for the average spectrum, but many of the resulting parameters were unconstrained. Since no spectral variability is detected above $5 \mathrm{keV}$ (see Fig. 3), we kept both the normalization and the temperature of the hot plasma fixed at the values found for the average spectrum. No substantial change in the parameters is found from Table 3 except for the partial absorber, implying that above $0.5 \mathrm{keV}$, this component is responsible for the modulation. On the other hand, we are unable to identify the components responsible for the soft $\mathrm{X}$-ray pulsation.

\section{Discussion}

Our XMM-Newton observation of UU Col has revealed new properties of this poorly studied magnetic system.

\subsection{The pulsation properties}

The X-ray variability is dominated by the $863.3 \mathrm{~s}$ pulsation ascribed to the WD rotational period by Burwitz et al. (1996). The detection for the first time of a weak (9\%) 935.4 s X-ray periodicity, consistent with the $\omega-\Omega$ orbital sideband, indicates that two accretion modes exist (see Hellier 1995; Norton et al. 1996). In fact, the presence of a dominant (full amplitude 75\%) spin pulsation implies that accretion occurs mainly via a disc because the material circulating in the disc loses memory of the orbital motion; but an additional beat $(\sim 20 \%)$ variability indicates that part of the accreting material also flows directly towards the WD without passing through the disc (disc-overflow). A similar behaviour was observed in a few other IPs like TX Col (Norton et al. 1997) and FO Aqr (Beardmore et al. 1998).
Table 3. Spectral parameters as derived from fitting the EPIC PN spectrum at maximum and minimum of spin pulsation using Model A in Table 2 .

\begin{tabular}{|c|c|c|}
\hline & Pulse maximum & Pulse minimum \\
\hline $\begin{array}{c}F_{2-10} \\
{\left[10^{-12} \mathrm{erg} \mathrm{cm}^{-2} \mathrm{~s}^{-1}\right]}\end{array}$ & $1.90 \pm 0.02$ & $1.74 \pm 0.03$ \\
\hline$N_{\mathrm{H}}\left[10^{23} \mathrm{~cm}^{-2}\right]$ & $1.01_{-0.34}^{+0.29}$ & $1.33_{-0.27}^{+0.41}$ \\
\hline $\operatorname{Cov}_{F}$ & $0.49_{-0.02}^{+0.03}$ & $0.56 \pm 0.03$ \\
\hline$k T_{\mathrm{BB}}[\mathrm{keV}]$ & $50_{-10}^{+0.02}$ & $51_{-10}^{+\infty}$ \\
\hline$C_{\mathrm{BB}}\left[10^{-5}\right]$ & $1.25_{-0.15}^{+1.03}$ & $1.13_{-\infty}^{+1.07}$ \\
\hline$k T_{1}[\mathrm{keV}]$ & $0.16_{-0.04}^{+0.75}$ & $0.17_{-0.03}^{+0.04}$ \\
\hline$C_{1}\left[10^{-4}\right]$ & $\begin{array}{l}3.4_{-1.4}^{+4.04} \\
\text { +4.2 }\end{array}$ & $3.0_{-0.9}^{+11.03}$ \\
\hline$A_{7}^{a}$ & 0.39 & 0.39 \\
\hline$k T_{2}^{a}[\mathrm{keV}]$ & 11 & 11 \\
\hline$C_{2}^{a}\left[10^{-4}\right]$ & 19.04 & 19.04 \\
\hline$\chi_{v}^{2}\left(\chi^{2} /\right.$ d.o.f. $)$ & $0.90(127 / 141)$ & $1.01(111 / 110)$ \\
\hline
\end{tabular}

The power spectrum also shows substantial power at the second harmonic of the spin frequency $(3 \omega)$. A similar feature was also observed in the power spectrum of V709 Cas (Norton et al. 1999; de Martino et al. 2001) and interpreted as the contribution of two asymmetric accreting poles. However, energy-resolved power spectra show that in UU Col the $3 \omega$ frequency dominates below $0.5 \mathrm{keV}$, implying that the accretion pattern and its associated emission properties are very complex. Furthermore, the presence of an orbital modulation only in the soft X-ray range implies that the responsible region is not strictly related to the one producing the hard X-ray emission. This aspect is explored below.

The complex energy dependence of the spin pulse suggests contribution from multiple components. No modulation is detected above $5 \mathrm{keV}$ indicating no influence from the hottest postshock regions on the pulsation, but between $0.5-5 \mathrm{keV}$ the spin is probably due to photo-electric absorption. This behaviour is observed in most IPs (Norton \& Watson 1989; de Martino et al. 2001, 2004). The hardening at spin minimum and the identification of a dense $\left(10^{23} \mathrm{~cm}^{-2}\right)$ absorber, covering about $50 \%$ of the X-ray source, is consistent with the standard accretion curtain scenario of IPs (Rosen et al. 1988), where the material from the accretion disc is captured by the magnetic field lines flowing towards the WD poles in an arc-shaped curtain. The complexity and inhomogeneity of the absorber in IPs was first recognised by Mukai et al. (1994).

The appearance of an additional maximum in the soft $0.2-0.5 \mathrm{keV}$ band at the minimum of the hard pulse indicates that, besides the main accreting pole, that is active in both soft and hard X-ray ranges, there is a substantial soft X-ray contribution from the secondary pole. It therefore appears that the soft $\mathrm{X}$-rays comprise contributions from both the primary and secondary poles. This behaviour is also seen in the soft IP V405 Aur (Evans \& Hellier 2004), though with its individual differences, but not in the other IPs of this small group of soft X-ray systems (de Martino et al. 2004). The soft spin pulse in V405 Aur is double-peaked, while the light curve in UU Col has three maxima indicating that the accretion geometry in the latter is more complex.

The presence of a soft X-ray orbital modulation and the strong soft X-ray contribution from the secondary pole suggests that accretion flowing directly from the stream preferentially impacts onto this pole. Also, that the spin light curve has 
three peaks might suggest an asymmetry in the overflow impact regions. From our spectral analysis at maximum and at minimum of the spin pulsation, we unfortunately cannot establish whether the secondary pole is dominated by the black-body soft $\mathrm{X}$-ray emission or the optically thin cool component. However, we have found that the UV and optical spin pulsations are antiphased with respect to the hard X-ray pulse and that they show a broad maximum and strong colour dependence. This implies that UV/optical rotational modulation does not originate in the accretion curtain above the main accreting pole but it arises from the heating of a large area of the WD surface at the secondary pole. From the amplitudes of UV and $B$ band spin modulations (15\% and $6 \%$, respectively) we derive a colour temperature of $\sim 31000 \mathrm{~K}$ assuming a black-body emission of the region producing the UV/optical spin modulated flux. Furthermore, the pulsed fluxes at the effective wavelengths of the $U V M 2$ and $B$ filters imply a radius of $\sim 7 \times 10^{7} D_{100 \mathrm{pc}} \mathrm{cm}$, for a WD with $\log g=8.0$ and $T_{\text {eff }}=30000 \mathrm{~K}$, where $D_{100}$ pc is the distance in units of $100 \mathrm{pc}$. Based on the $K$-band surface brightness, Burwitz et al. (1996) estimated a lower limit of $740 \mathrm{pc}$ for the distance of UU Col, which would imply $R_{\text {spot }} \geq 5.2 \times 10^{8} \mathrm{~cm}$, and hence a relatively large spot of the WD surface. It may therefore occur that the two-mode accretion onto the WD involves both poles with the secondary emitting radiation mainly at lower energies.

\subsection{The accretion flow properties}

The X-ray spectral analysis reveals the presence of multiple components: a hot optically thin plasma at $11 \mathrm{keV}$, which is visible throughout the spin cycle. The emission measure derived for this component is $E M_{\text {hot }}=2.3 \times 10^{53} D_{100 \mathrm{pc}}^{2} \mathrm{~cm}^{-3}$ and its bolometric luminosity is $6.4 \times 10^{30} D_{100 \mathrm{pc}}^{2} \mathrm{erg} \mathrm{s}^{-1}$. A multi-temperature power-law structure of the post-shock region does not seem to be required though a cooler thin emission at $0.18 \mathrm{keV}$ is clearly present. This low-temperature plasma is also detected in V405 Aur (Evans \& Hellier 2004). We derive an emission measure $E M_{\text {cool }}=2.9 \times 52 D_{100 \mathrm{pc}}^{2} \mathrm{~cm}^{-3}$ for this component and a bolometric luminosity of $1.1 \times 10^{30} D_{100 \mathrm{pc}}^{2} \mathrm{erg} \mathrm{s}^{-1}$, hence $\sim 8$ and $\sim 6$ times smaller than those of the hot, optically thin emission, respectively. It is likely that this component originates much closer to the WD surface than the hotter region. The strong OVIII and OVII lines detected in the RGS spectra, which map the low temperature plasma conditions particularly, are well accounted for by the cool thin plasma component. The flux ratio of these lines indeed indicates a temperature of $0.2_{-0.03}^{+0.1} \mathrm{keV}$. The OVII He-like features tentatively identified as the resonant and forbidden components give a ratio $r / f \sim 28$ suggesting a collision-dominated plasma (Porquet et al. 2001). We are unable to derive an estimate of the density because of the weakness of the features in the RGS spectra.

The X-ray spectrum also shows a black-body soft X-ray component at $50 \mathrm{eV}$, similar to that found for PQ Gem and V405 Aur, the two other bright soft X-ray IPs (de Martino et al. 2004; Evans \& Hellier 2004). Its bolometric luminosity is $1.3 \times 10^{30} D_{100 \mathrm{pc}}^{2} \mathrm{erg} \mathrm{s}^{-1}$. The emitting area of this component is $a_{\mathrm{BB}}=2.0 \times 10^{11} D_{100 \mathrm{pc}}^{2} \mathrm{~cm}^{2}$. At the minimum distance of $740 \mathrm{pc}$, this gives $r_{\mathrm{BB}} \sim 2 \times 10^{6} \mathrm{~cm}$, which is much smaller than the radius of the UV/optical emitting region. The ratio of bolometric fluxes between the soft X-ray, black-body, and hard X-ray components is only 0.20 and hence lower than that estimated by Burwitz et al. (1996), who assumed a simple model consisting of a black-body at $25 \mathrm{eV}$ and a thermal
Bremsstrahlung at $20 \mathrm{keV}$. However, we note that using this same simple model, we derive a ratio $F_{\mathrm{BB}} / F_{\text {th. }} \sim 13$. It is therefore clear that previous determination of the energy balance is subject to strong revisions due to proper determination of the spectral components.

We have seen that the secondary pole radiates mainly at low energies, and it is responsible for the orbital modulation in the soft X-rays. This might suggest that this pole is fed predominantly by material not circulating in the disc. To estimate the accretion luminosity, we then take the different spectral components into account. Hence, assuming that the accretion luminosity is emitted in the hard, soft X-rays, and UV/optical wavelengths, $L_{\mathrm{acc}}=L_{\mathrm{BB}}+L_{\mathrm{th}}+L_{\mathrm{UV} / \mathrm{Opt}}=9.5 \times 10^{30} D_{100 \mathrm{pc}}^{2} \mathrm{erg} \mathrm{s}^{-1}$, where we include both optically thin components in the bolometric flux computation, we derive an accretion rate of $1.5 \times$ $10^{-12} D_{100 \mathrm{pc}}^{2} M_{\odot} \mathrm{yr}^{-1}$ for a WD mass of $0.6 M_{\odot}$. At the minimum distance of $740 \mathrm{pc}$, the mass accretion rate is much lower than the secular rate predicted for a $\mathrm{CV}$ with a $3.5 \mathrm{~h}$ orbital pe$\operatorname{riod} \dot{M}=2.0 \times 10^{-11} P_{\Omega, \mathrm{hr}}^{3.7} M_{\odot} \mathrm{yr}^{-1}$ (see Warner 1995), unless the distance is extremely large $(\sim 3.5 \mathrm{kpc})$. It therefore seems that UU Col is a soft X-ray IP with a low mass-accretion rate. The lower limit to the distance and the high latitude would put this system well above the galactic disc and about 2-3 scale heights above the one of the CV population. This makes it unlikely that UU Col belongs to the disc population. If it is a rare case of a halo $\mathrm{CV}$, this might explain its very low-mass accretion rate.

The ratio of spin-to-orbit periods is $P_{\omega} / P_{\Omega}=0.07$ close to 0.1 , which is the typical value of the period ratios of IPs. Though we detected that accretion also occurs directly from the stream, the bulk of material is accreted via a disc. We then investigated the spin equilibrium state by first evaluating the corotation radius, defined as the radius at which the magnetic field rotates at the Keplerian frequency: $R_{\mathrm{co}}=\left(G M_{\mathrm{WD}} P_{\omega}^{2} / 4 \pi^{2}\right)^{1 / 3}$. For a rotational period of $863 \mathrm{~s}$ and $M_{\mathrm{WD}}=0.6 M_{\odot}, R_{\mathrm{co}}=$ $1.2 \times 10^{10} \mathrm{~cm}$. The condition for accretion requires $R_{\mathrm{mag}} \leq R_{\mathrm{co}}$ with $R_{\text {mag }}=5.5 \times 10^{8}\left(M_{\mathrm{WD}} / M_{\odot}\right)^{1 / 7} R_{9}^{-2 / 7} L_{33}^{-2 / 7} \mu_{30}^{4 / 7} \mathrm{~cm}$, where $R_{9}$ is the WD radius in units of $10^{9} \mathrm{~cm}, L_{33}$ is the luminosity in units of $10^{33} \mathrm{erg} \mathrm{s}^{-1}$, and $\mu_{30}$ is the magnetic moment in units of $10^{30} \mathrm{G} \mathrm{cm}^{3}$. Using our mass accretion rate determination, we then find $\mu \leq 2 \times 10^{31} D_{100 \mathrm{pc}} \mathrm{G} \mathrm{cm}^{3}$. Norton et al. (2004) derive a magnetic moment of $6.5 \times 10^{32} \mathrm{G} \mathrm{cm}^{3}$ for UU Col by applying their magnetic accretion model and assuming it is in spin equilibrium. Their estimate of the magnetic moment is about 4 times larger than our upper limit for the minimum distance of $740 \mathrm{pc}$, but we note that they assume an accretion rate corresponding to the secular value predicted for its orbital period, which we found is too high for UUCol. Hence UU Col appears to possess a weak magnetic field WD accreting at a relatively low rate. The condition of spin equilibrium is attained when $R_{\text {circ }} \sim R_{\text {co }}$, where $R_{\text {circ }}$ is the radius at which material from the stream leaving the secondary star at the inner Lagrangian point $L_{1}$ circulates in a Keplerian orbit around the WD, defined as $R_{\text {circ }} \simeq 4 \pi^{2} P_{\Omega}^{-2} b^{4}\left(G M_{\mathrm{WD}}\right)^{-1}$, with $b$ the distance of $L_{1}$ from the WD. Assuming a typical mass ratio $q=0.5$, we derive $R_{\text {circ }} \sim 1.5 \times 10^{10} \mathrm{~cm}$. Hence UU Col is close to its equilibrium value.

\section{Conclusions}

Based on the first XMM-Newton observations, and complemented by UV and optical simultaneous photometry, our X-ray study of UUCol has shown interesting new properties of this 
poorly studied faint soft X-ray IP. These can be summarised as follows:

- The X-ray $(0.2-15 \mathrm{keV})$ variability is dominated by the $863 \mathrm{~s}$ spin periodicity but a weak variability is also detected at the $935 \mathrm{~s}$ beat period. This indicates two accretion modes operating in this system with the bulk $(\sim 80 \%)$ of accretion occurring via a disc and with a small fraction $(\sim 20 \%)$ accreting directly from the stream.

- The spin pulse shows a complex energy dependence revealing the presence of the secondary pole that emits mainly below $0.5 \mathrm{keV}$. At intermediate energies between $0.5-5 \mathrm{keV}$, the spin pulse is probably due to photoelectric absorption. Above $5 \mathrm{keV}$ no modulation is observed indicating that the hottest post-shock regions of the main accreting pole are viewed throughout the spin cycle.

- We also detected an orbital periodicity below $0.5 \mathrm{keV}$ without any counterpart in the hard X-rays. The spin pulse shape at maximum and minimum of the orbital cycle suggests a dominant contribution from the secondary pole.

- The UV and optical pulses show a broad maximum at the minimum of the hard X-ray pulsation, revealing that they arise from a large region at the WD surface close to the secondary pole.

- The X-ray spectrum of UUCol is complex, consisting of a soft X-ray black-body component at $\sim 50 \mathrm{eV}$ and two optically thin emissions at $0.2 \mathrm{keV}$ and $11 \mathrm{keV}$, highly absorbed by dense $\left(10^{23} \mathrm{~cm}^{-2}\right)$ material partially $(50 \%)$ covering the X-ray source. The RGS spectra reveal strong OVIII and OVII lines confirming the presence of the cooler, optically thin emitting region.

- The ratio of soft-to-hard bolometric fluxes is not high $\left(F_{\mathrm{BB}} / F_{\text {th }}=0.2\right)$, consistent with the prediction of the standard model of accretion shocks.

- From the X-ray, UV, and optical luminosities we infer a mass accretion rate of $1.5 \times 10^{-12} D_{100 \mathrm{pc}}^{2} M_{\odot} \mathrm{yr}^{-1}$, lower than the secular value expected for its $3.5 \mathrm{~h}$ orbital period.

- The WD in UUCol is found to possess a low magnetic moment and to rotate at its equilibrium value. Therefore,
UU Col appears to be different from the other two wellstudied soft X-ray bright IPs PQ Gem and V405 Aur, which instead possess stronger magnetic fields. Hence UUCol might not evolve into a moderate field-strength polar, meaning that the soft $\mathrm{X}$-ray systems still remain an enigmatic small group of IPs.

Acknowledgements. D.D.M. acknowledges financial support by the Italian Minister of University and Research (MIUR) and the Italian Space Agency (ASI) under contract I/023/05/0.

\section{References}

Anders, E., \& Grevesse, N. 1989, GeCoA, 53, 197

Beardmore, A. P., Mukai, K., Norton, A. J., Osborne, J. P., \& Hellier, C. 1998, MNRAS, 297, 337

Burwitz, V., \& Reinsch, K. 2001, AIPC, 599, 522

Burwitz, V., Reinsch, K., Beuermann, K., \& Thomas, H.-C. 1996, A\&A, 310, L25

Cumming, A. 2002, MNRAS, 333, 589

Evans, P. A., \& Hellier, C. 2004, MNRAS, 353, 447

de Martino, D., Matt, G., Mukai, K., et al. 2001, A\&A, 377, 499

de Martino, D., Matt, G., Belloni, T., et al. 2004, A\&A, 415, 1009

den Herder, J. W., Brinkman, A. C., Kahn, S. M., et al. 2001, A\&A, 365, L7

Haberl, F., \& Motch, C. 1995, A\&A, 297, L37

Haberl, F., Motch, C., \& Zickgraf, F.-J. 2002, A\&A, 387, 201

Hellier, C. 1995, ASP Conf. Ser., 85, 185

Jansen, F., Lumb, D., Altieri, B., et al. 2001, A\&A, 365, L1

Mason, K. O., Breeveld, A., Much, R., et al. 2001, A\&A, 365, L36

Mukai, K., Ishida, M., \& Osborne, J. P. 1994, PASJ, 46, L87

Norton, A. J., \& Watson, M. G. 1989, MNRAS, 237, 715

Norton, A. J., Beardmore, A. P., \& Taylor, P. 1996, MNRAS, 280, 937

Norton, A. J., Hellier, C., Beardmore, A. P., et al. 1997, MNRAS, 289, 362

Norton, A. J., Beardmore, A. P., Allan, A., \& Hellier, C., 1999, A\&A, 347, 293

Norton, A. J., Wynn, G. A., \& Somerscales, R. V. 2004, ApJ, 614, 349

Porquet, D., Mewe, R., Dubau, J., et al. 2001, A\&A, 376, 1113

Rosen, S. R., Mason, K. O., \& Cordova, F. A. 1988, MNRAS, 231, 549

Strüder, L., Briel, U., Dennerl, K., et al. 2001, A\&A, 365, L18

Staude, A., Schwope, A. D., Krumpe, M., et al. 2003, A\&A, 406, 253

Turner, M. J. L., Abbey, A., Arnaud, M., et al. 2001, A\&A, 365, L27

Warner, B. 1995, Cataclsymic Variables (Cambridge: Cambridge Univ. Press)

Wilms, J., Allen, A., \& McCray, R. 2000, ApJ, 542, 914

Woelk, U., \& Beuermann, K. 1992, A\&A, 256, 498 